\title{
RTA Beam Dynamics Experiments: Limiting Cumulative Transverse Instability Growth in a Linear Periodic System
}

T. Houck, S. Lidia, G. Westenskow

This article was submitted to Advances Accelerator Concepts Workshop, Santa Fe, NM June 10-16, 2000

\section{June 10, 2000}

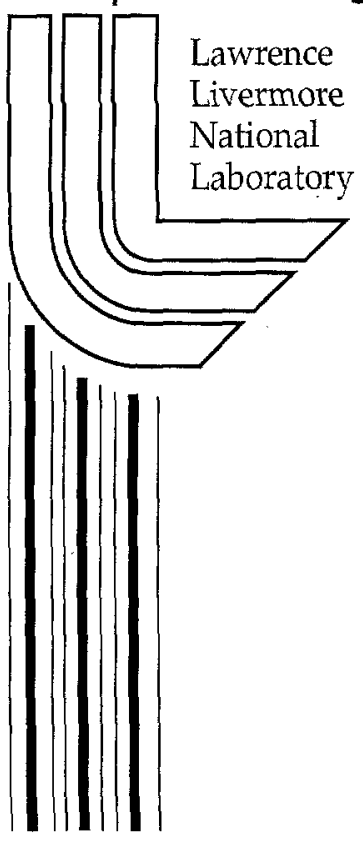




\section{DISCLAIMER}

This document was prepared as an account of work sponsored by an agency of the United States Government. Neither the United States Government nor the University of California nor any of their employees, makes any warranty, express or implied, or assumes any legal liability or responsibility for the accuracy, completeness, or usefulness of any information, apparatus, product, or process disclosed, or represents that its use would not infringe privately owned rights. Reference herein to any specific commercial product, process, or service by trade name, trademark, manufacturer, or otherwise, does not necessarily constitute or imply its endorsement, recommendation, or favoring by the United States Government or the University of California. The views and opinions of authors expressed herein do not necessarily state or reflect those of the United States Government or the University of California, and shall not be used for advertising or product endorsement purposes.

This is a preprint of a paper intended for publication in a journal or proceedings. Since changes may be made before publication, this preprint is made available with the understanding that it will not be cited or reproduced without the permission of the author.

This report has been reproduced directly from the best available copy.

Available electronically at http://www.doc.gov/bridge

Available for a processing fee to U.S. Department of Energy

And its contractors in paper from

U.S. Department of Energy

Office of Scientific and Technical Information

P.O. Box 62

Oak Ridge, TN 37831-0062

Telephone: (865) 576-8401

Facsimile: (865) 576-5728

E-mail: reports@adonis.osti.gov

Available for the sale to the public from

U.S. Department of Commerce

National Technical Information Service

5285 Port Royal Road

Springfield, VA 22161

Telephone: (800) 553-6847

Facsimile: (703) 605-6900

E-mail: orders@ntis.fedworld.gov

Online ordering: http://www.ntis.gov/ordering.htm

\section{OR}

Lawrence Livermore National Laboratory

Technical Information Department's Digital Library

http://www.llnl.gov/tid/Library.html 


\title{
RTA Beam Dynamics Experiments: Limiting Cumulative Transverse Instability Growth In A Linear Periodic System*
}

\author{
Tim Houck, Steve Lidia**, and Glen Westenskow \\ Lawrence Livermore National Laboratory, 7000 East Avenue, L-645 \\ Livermore. CA 94550-9234 \\ ** Lawrence Berkeley National Laboratory, I Cyclotron Road, Mailstop 47-I12 \\ Berkeley, CA 94720
}

\begin{abstract}
A bstract. A critical issue for a Two-Beam accelerator based upon extended relativistic klystrons is controlling the cumulative dipole instability growth We describe a theoretical scheme to reduce the growth from an exponential to a more manageable linear rate, and a new experiment to test this concept. The experiment utilizes a 1-MeV, 600-Amp, 200-ns electron beam and a short beamline of periodically spaced RF dipole pillbox cavities and solenoid magnets for transport. Descriptions of the RTA injector and the planned beamline are presented, followed by theoretical studies of the beam transport and dipole mode growth.
\end{abstract}

\section{INTRODUCTION}

A Lawrence Livermore National Laboratory (LLNL) and Lawrence Berkeley National Laboratory (LBNL) collaboration is studying the application of induction accelerator technology to the generation of microwave power. We refer to this scheme of power generation as the Relativistic Klystron Two-Beam Accelerator (RK-TBA) [1]. This scheme is considered a TBA approach as the extraction of microwave power is distributed along a drive beam parallel to the high-energy RF linear accelerator. The RK designation indicates that the power is generated by the interaction of the relativistic modulated drive beam with resonant structures similar to those used in a conventional klystron.

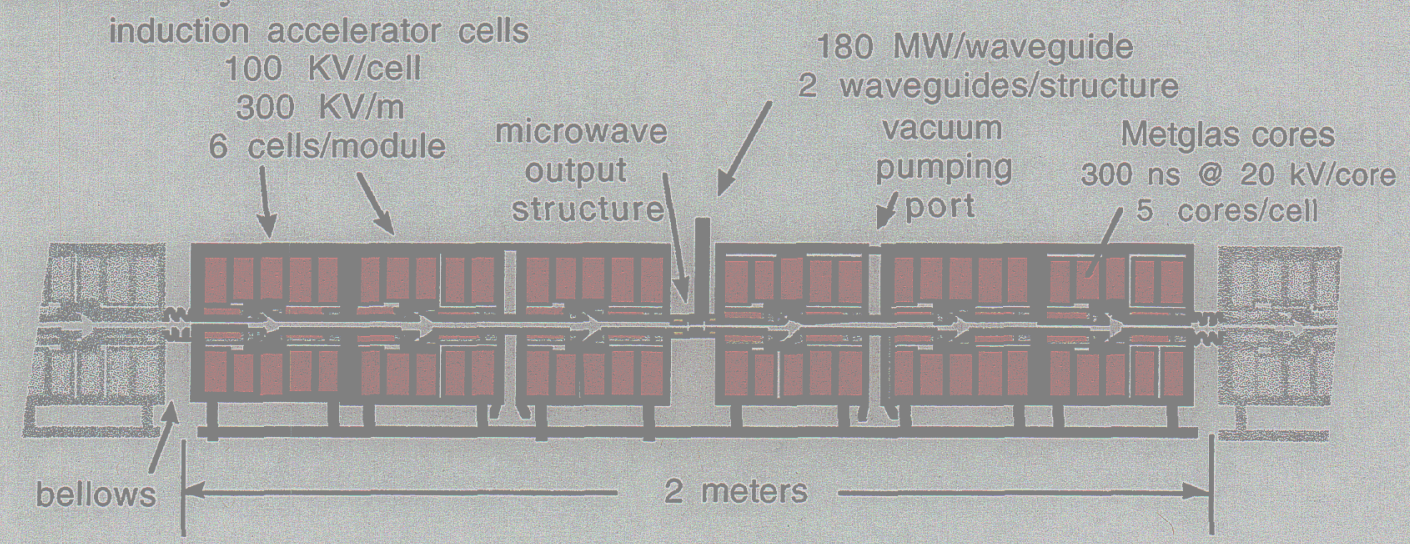


FIGURE 1. Illustration of a repeating module that comprises the extraction section of a RK.

The primary advantage of TBA concepts is that the conversion of drive beam power to microwave power can be highly efficient $(>90 \%)$. This efficiency is realized by distributing the power extraction over an extended length. The interest in RK-TBA's is that induction accelerators are efficient at producing very high power electron beams. Present induction accelerators operate at currents of several kiloamperes and accelerate the beam to $10^{\prime} \mathrm{s}$ of $\mathrm{MeV}$ for beam power of 100's GW [2]. The induction accelerator can realize improved efficiency at converting wall plug power into beam power by replacing the standard electromagnet solenoids for beam transport with permanent magnets. Even higher efficiency can be attained if the induction cells are used as highvoltage step up transformers driven by a relative low voltage $(\sim 20 \mathrm{kV})$ pulsed power system. Present designs of a RK-TBA predict efficiency of about $40 \%$ in conversion of wall plug power into beam power [3], or a total wall plug to microwave power efficiency of about $36 \%$.

The main section of an RK where the microwave power is generated is comprised of many repeating modules as illustrated in Figure 1. Within each module, the induction cells replace the energy extracted from the electron beam by the microwave output structure. The efficiency of this process - extraction and reacceleration - is nearly $100 \%$. Not shown in Figure 1 are the beam generation and modulation sections and the final beam dump. Fixed energy losses in those processes have to be included in calculating the total beam energy to microwave conversion efficiency. Thus, it is imperative that the RK have many of the efficient extraction and reacceleration cycles to reduce the relative value of fixed losses with respect to the total energy transferred to the beam.

Several proof-of-concept experiments have been performed to demonstrate the viability of the RK-TBA concept. These experiments have shown the generation of collider-scale drive beam in induction linacs, production of high-quality, high-power microwaves from standing- and traveling-wave structures driven by induction accelerator beams, and multiple reacceleration and extraction cycles $[4,5]$. As will be described below, we are continuing to perform experiments to study specific physics and technology issues while constructing a prototype relativistic klystron.

\section{RTA FACILITY STATUS}

The RTA Facility at LBNL was established to study issues related to RK-TBA designs. The principle effort is the construction of a prototype RK to serve as a test bed for physics, engineering, and cost studies. We have completed the first major component of the prototype RK, the commissioning of the induction gun [6]. A schematic of the gun is shown in Figure 2. The induction cores are individually driven at $15 \mathrm{kV}$ as a demonstration of the type of pulsed power system proposed for a full scale RK. The 3.5" M-type dispenser cathode and electrodes are designed to produce a normalized edge emittance of $300 \pi \mathrm{mm}-\mathrm{mr}$. Currently we are performing some minor refurbishment to the gun and upgrades to the pulsed power system. Once this work is finished, we will re-characterize the beam and then begin a series of beam dynamics experiments (described below).

A second effort involves parameter and optimization studies of possible RK-TBA designs. We have considered microwave frequencies from X-band to $\mathrm{Ka}$-band [7] for powering colliders with center-of-mass energies up to 5-TeV. In support of the higher frequency designs, we participated in an experiment where an induction beam was modulated at $35 \mathrm{GHz}$ using a FEL and then transported through a resonant cavity to 
generate power [8]. This experiment was performed at CESTA with collaborators and support from the University of Bordeaux, LBNL, LLNL, and CERN. Additional experiments are planned.

We are also continuing to develop relevant induction accelerator technology in collaboration with other induction accelerator programs at LLNL, LBNL, and Los Alamos National Laboratory (LANL) as well as with small businesses [9]. A recent example is the testing of a High Gradient Insulator (HGI) [10] on LLNL's ETA-II accelerator. The HGI is a multilayered construction of alternating insulator and conductor material that has shown great ability in holding off electrical fields. The HGI has also been used in induction cell designs to produce lower impedance. The test placed an HGI in an actual induction cell (modified ETA-II cell) under electrical field stress in excess of $100 \mathrm{kV} / \mathrm{cm}$ during accelerator operations with a $2-\mathrm{kA}$ beam. The sucessful demonstration of the insulator under realistic conditions was a major milestone towards the incorporation of HGI technology into operating accelerators.

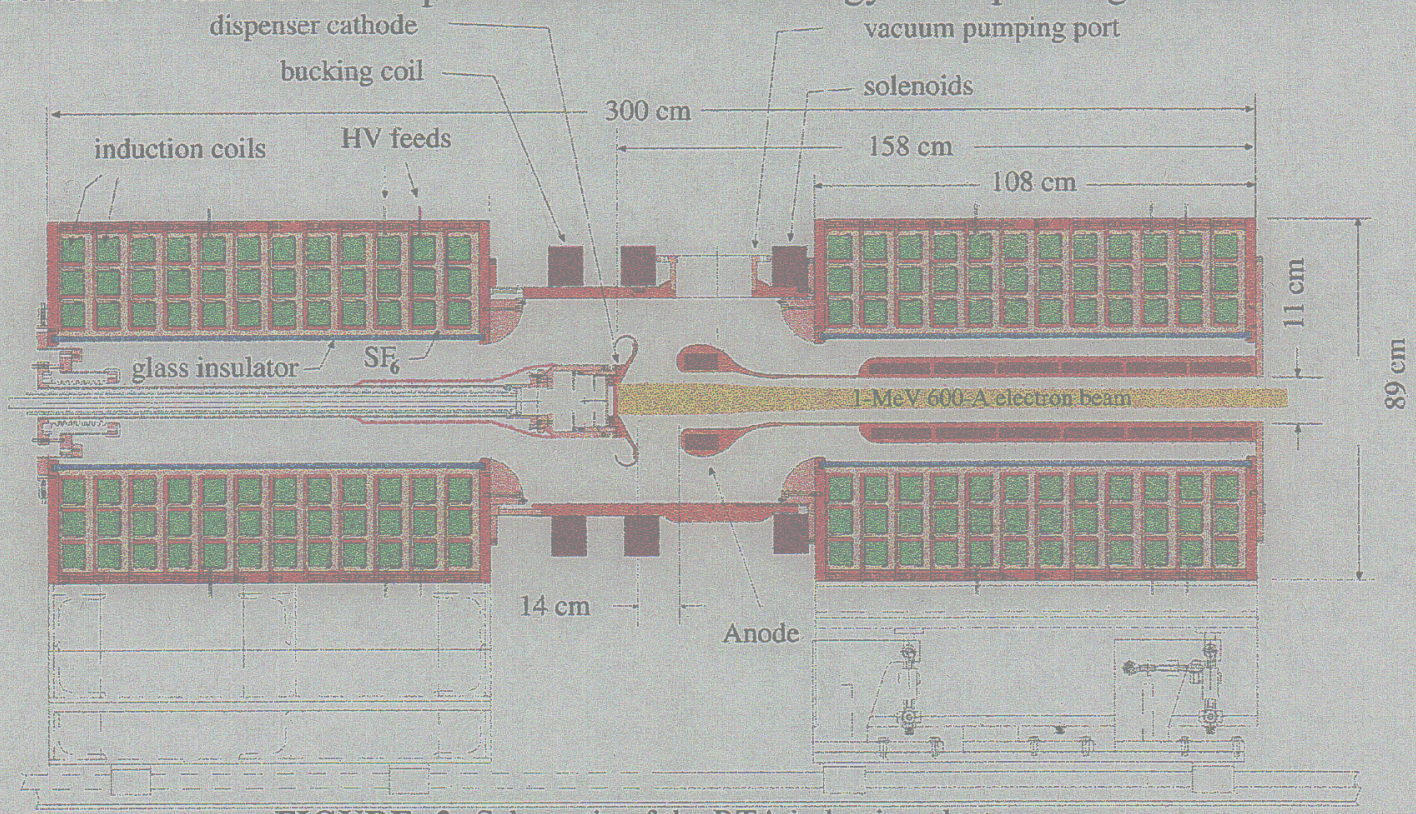

FIGURE 2. Schematic of the RTA induction electron gun.

An important issue for the RK-TBA is the conversion of wall plug to microwave power efficiency. Most of the components of an RK can be independently tested to determine efficiency, cost, and lifetime - e.g. pulsed power components and induction cells. However, the number of extraction cavities determines the overall efficiency of the RK, and this number is limited by beam dynamics. We expect the prototype RK to be able to address all the relevant beam dynamics issues. However, prior to the completion of the prototype, we intend to do a series of smaller proof of concept experiments to study specific beam dynamics issues.

\section{BEAM DYNAMICS ISSUES}

The ultimate efficiency of a RK is determined by the induction beam dynamics i.e. the number of extraction structures that the beam can transit. We have identified three critical areas of beam dynamics that must be understood. The first involves maintaining the longitudinal modulation of the beam or "RF bucket" structure. In the drifts between output structures, space charge forces will cause the beam to lengthen in 
phase space, i.e., "debunch". If this effect is not corrected, the RF current (Fourier component of the beam at the modulation frequency) will decrease resulting in a decrease in the microwave power that can be extracted. Inductively detuning the output structures, similar to the penultimate cavity in conventional klystrons, can counter the space charge forces. The requirement for long-term longitudinal stability is reestablishing the initial longitudinal charge distribution at the end of a synchrotron period. Computer simulations have shown that with proper detuning, the RF current can be maintained over the 150 output structures envisioned for a full scale RK-TBA.

The other issues involve transverse instabilities. The beam will excite dipole modes in the induction cell accelerating gaps as well as in the resonant output structures. The induction cell accelerating gaps can be severely damped with $\mathrm{RF}$ absorbers for all resonant modes since the applied voltage pulse is quasi-static compared to the resonant frequencies. In addition, the natural energy spread over the RF bucket contributes to phase mixed, or Landau, damping. The combination of RF absorbers and energy spread is expected to maintain the transverse instability due to the dipole modes in the accelerating gaps at acceptable levels.

The resonant output structures present a more difficult transverse instability issue. The fundamental mode must couple sufficiently with the beam to extract the required energy. Various techniques exist to damp higher order modes in both output and accelerating structures. However, the permanent magnet focusing system envisioned for an RK-TBA allows the application of a new technique that we refer to as the Betatron Node Scheme.

Transverse beam instability theory is well developed and the exponential growth predicted is supported by experiment. However, the standard theoretical approach assumes that the discrete cavities interacting with the beam are closely spaced compared to the betatron wavelength due to the focusing system. Our design for an RK-TBA system requires strong focusing to maintain the required beam radius and a constant average energy over each extraction/reacceleration cycle. This combination leads to spacing between output structures of one betatron wavelength and the basic assumption of the standard theoretical approach does not hold.

An alternative approach to studying the transverse instability uses transfer matrices [11]. Assuming a monoenergetic beam and a thin cavity, Equations (1) through (3) indicate the salient parts of this theory. Equation (1) represents the transverse momentum change an electron receives passing through the cavity. $R$ is an integral operator that accounts for the part of the beam that has already passed through the cavity. The first matrix on the RHS of Equation (2) is then the transfer matrix for the beam going through the cavity. For a sufficiently thin cavity, the transverse position does not change. Only the momentum is affected. The second matrix represents the betatron motion of the electrons between cells where $\theta$ is the phase advance. Thus, Equation (1) advances the position and momentum of electrons from the exit of on cavity to the exit of the following cavity. By repeatedly multiplying the two transfer matrices, the position and momentum at the exit of any cavity can be related to the initial conditions. For the situation where $\theta$ is constant for all sections and $\theta \ll 1$, the series of matrix multiplications can be shown to yield the same expected exponential growth as the more standard approach.

$$
\begin{gathered}
\Delta p_{\perp}=R x \\
{\left[\begin{array}{c}
x \\
p_{\perp}
\end{array}\right]_{n+l_{\text {exit }}}=\left[\begin{array}{ll}
1 & 0 \\
R & 1
\end{array}\right]\left[\begin{array}{cc}
\cos \theta & \sin \theta / \omega \\
\omega \sin \theta & \cos \theta
\end{array}\right]\left[\begin{array}{c}
x \\
p_{\perp}
\end{array}\right]_{n_{\mathrm{exit}}}}
\end{gathered}
$$


For the case where $=2 \pi$ (or any integral multiple of $\pi$ ), the matrix multiplication is greatly simplified. The betatron motion returns the electrons to the original transverse position and momentum (oppositely directed for odd multiples of $\pi$ ). The multiplication involves only the matrix describing the effect of the cavity, and, as shown in Equation (3), this leads to a linear growth in the transverse instability.

$$
\left[\begin{array}{c}
x \\
p_{\perp}
\end{array}\right]_{n+1 \text { exit }}=\left[\begin{array}{ll}
1 & 0 \\
R & 1
\end{array}\right]^{n}\left[\begin{array}{c}
x \\
p_{\perp}
\end{array}\right]_{1_{\text {exit }}}=\left[\begin{array}{cc}
1 & 0 \\
n R & 1
\end{array}\right]\left[\begin{array}{c}
x \\
p_{\perp}
\end{array}\right]_{1_{\text {exit }}}
$$

There are many nonideal factors in a realistic accelerator including cavities of finite thickness and variation in phase advance due to energy and/or focusing errors. Parameter studies through computer simulations indicate that the transverse instability is significantly reduced for systems with reasonable variations in parameters. We intend to experimentally test the validity and robustness of the Betatron Node Scheme.

\section{BETATRON NODE SCHEME EXPERIMENT}

The basic elements involved in a test of the Betatron Node Scheme are: a set of devices that generate a localized transverse impedance, a tunable focusing and transport system, and diagnostics to measure the BBU mode signal on the beam as a function of time and distance along the beamline. A schematic for a possible beamline is shown in Figure 3. The localized impedances are generated in simple pillbox cavities, tuned so that the $\mathrm{TM}_{110}$ mode frequency matches the modulation of the beam; a series of solenoid magnets provide tunable focusing; and rf ("B-dot") loops ( $\mathrm{rf}$ BPM's) placed between cavities provide a means of collecting the dipole mode signal carried by the beam. We have built several sections of this beamline, using off-theshelf components wherever possible. Each section is one betatron wavelength long and is comprised of one pillbox cavity, a pumping port, a diagnostic; and three solenoids. A photograph of a single section is shown in Figure 4.

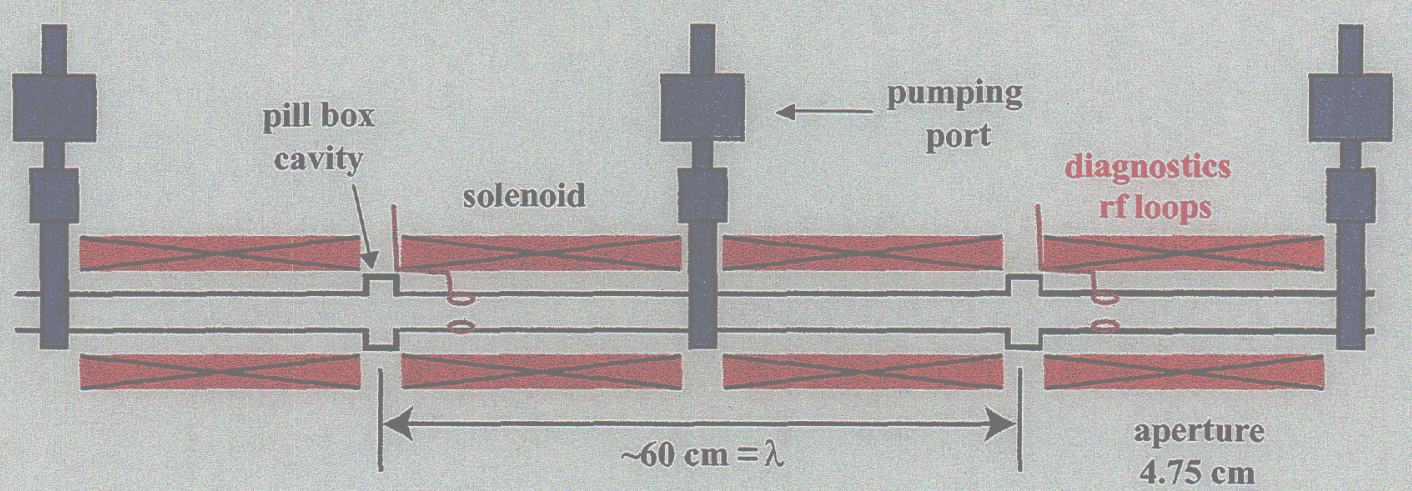

FIGURE 3. Schematic of the minimum beamline configuration required to demonstrate one period of the Betatron Node Scheme.

The designed solenoidal focusing field and expected beam envelope is indicated in Figure 5. The ten solenoids shown prior to the exit of the injector can be identified in the schematic of the electron gun (Figure 2). The next four solenoids are used to transport the beam through a transport region consisting of an inline gate valve and 
pumping station, and to match the beam into the repeating sections of the Betatron Node Scheme experiment. We will start with only two sections to verify transport calculations and diagnostic performance. Additional sections (up to a total of ten) and an initial driven tickler cavity will be added over the following months.

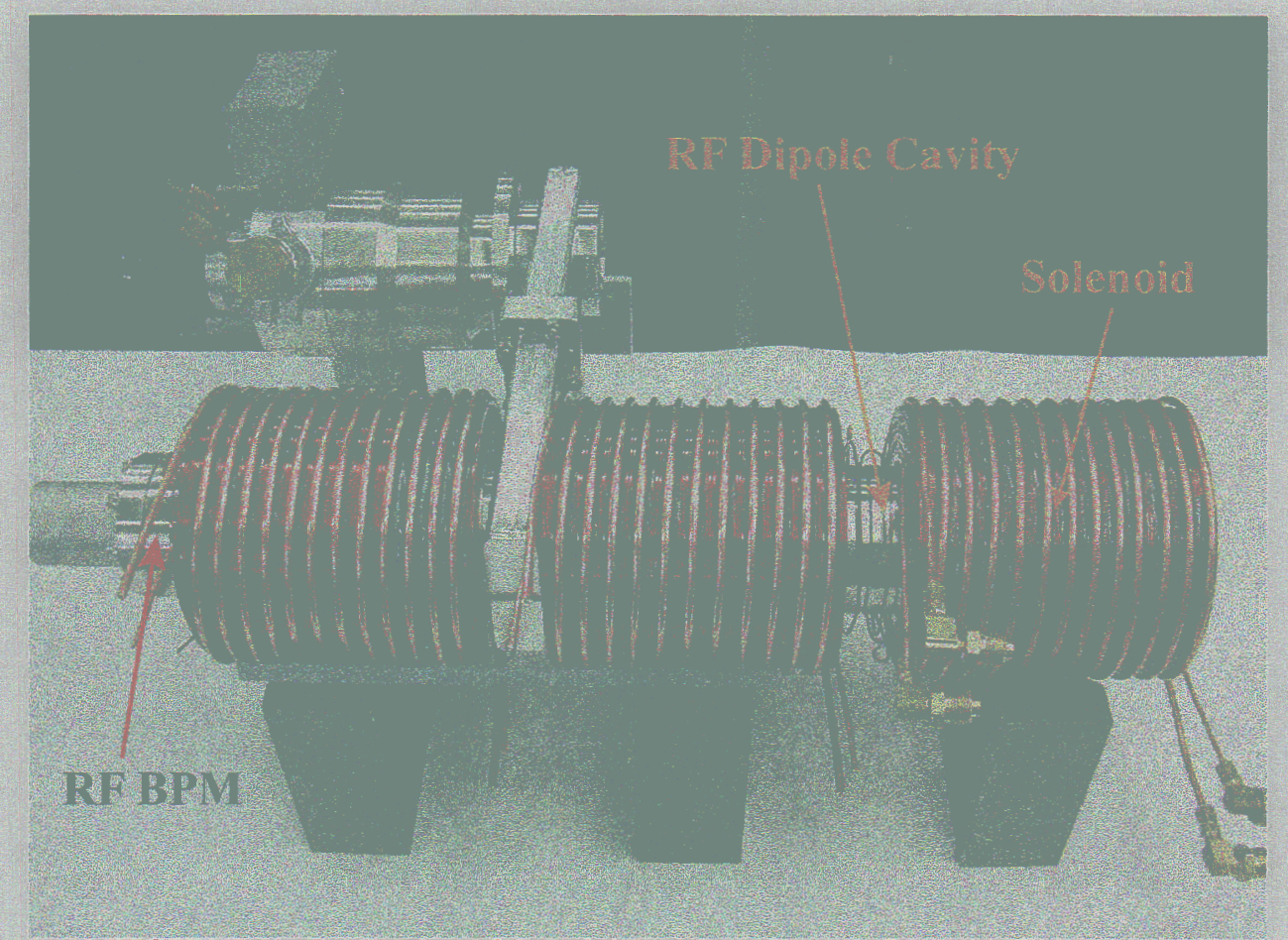

FIGURE 4. Realization of the minimum beamline configuration in hardware.
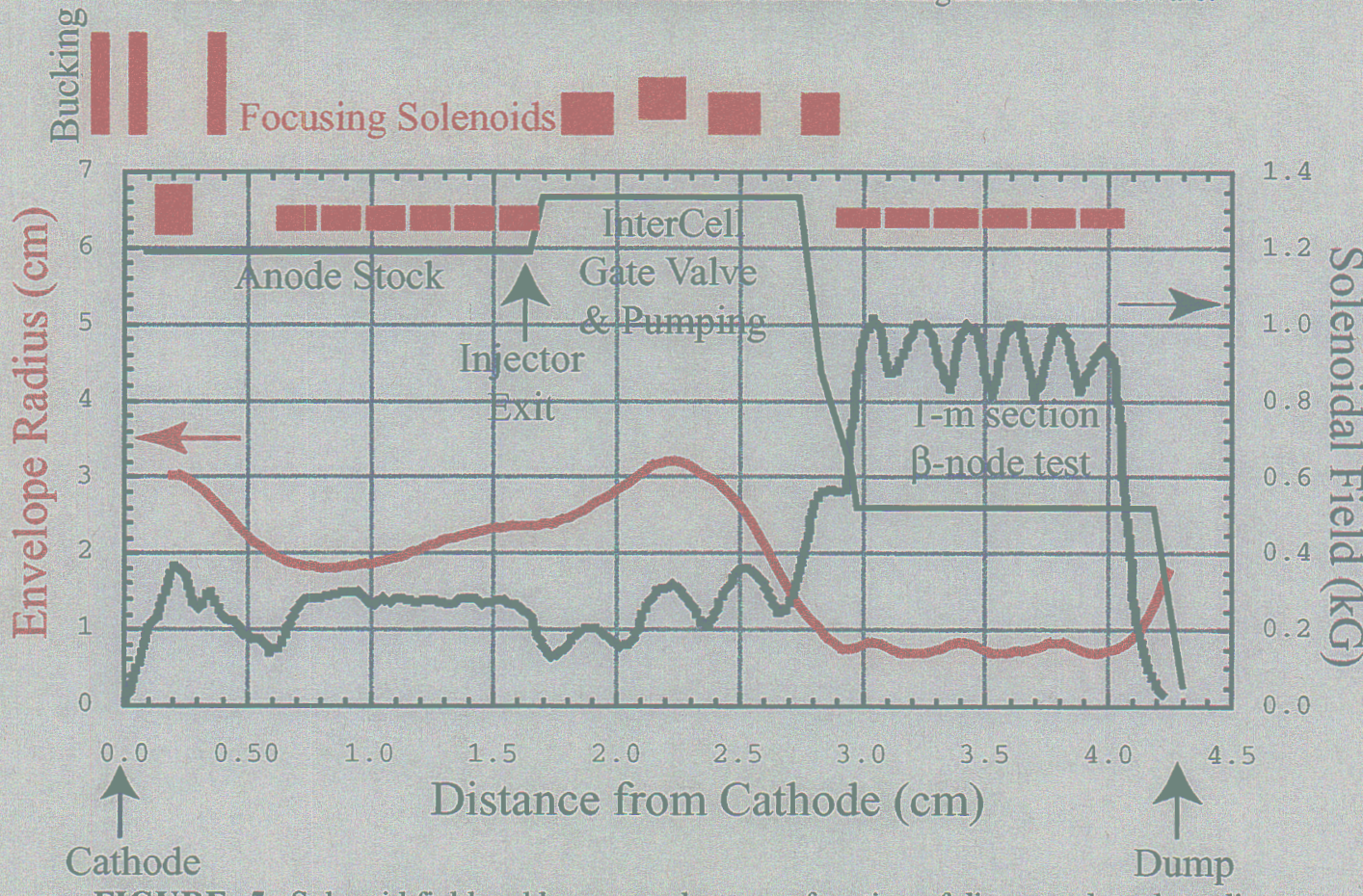

FIGURE 5. Solenoid field and beam envelope as a function of distance along beamline. 
Computer simulations of the increase in power measured by the RF diagnostics at the dipole mode frequency are shown in Figure 6 . Variations of $\pm 10 \%$ in the solenoidal field (betatron phase advance) from the optimum should produce several orders of magnitude increase in measured mode power after only a few sections. The graphs in Figure 6 indicate the maximum power expected during the main body of the beam ("flat top"). The temporal power variation during the pulse (not shown) is predicted to have different characteristics between under- and over-focused scenarios.
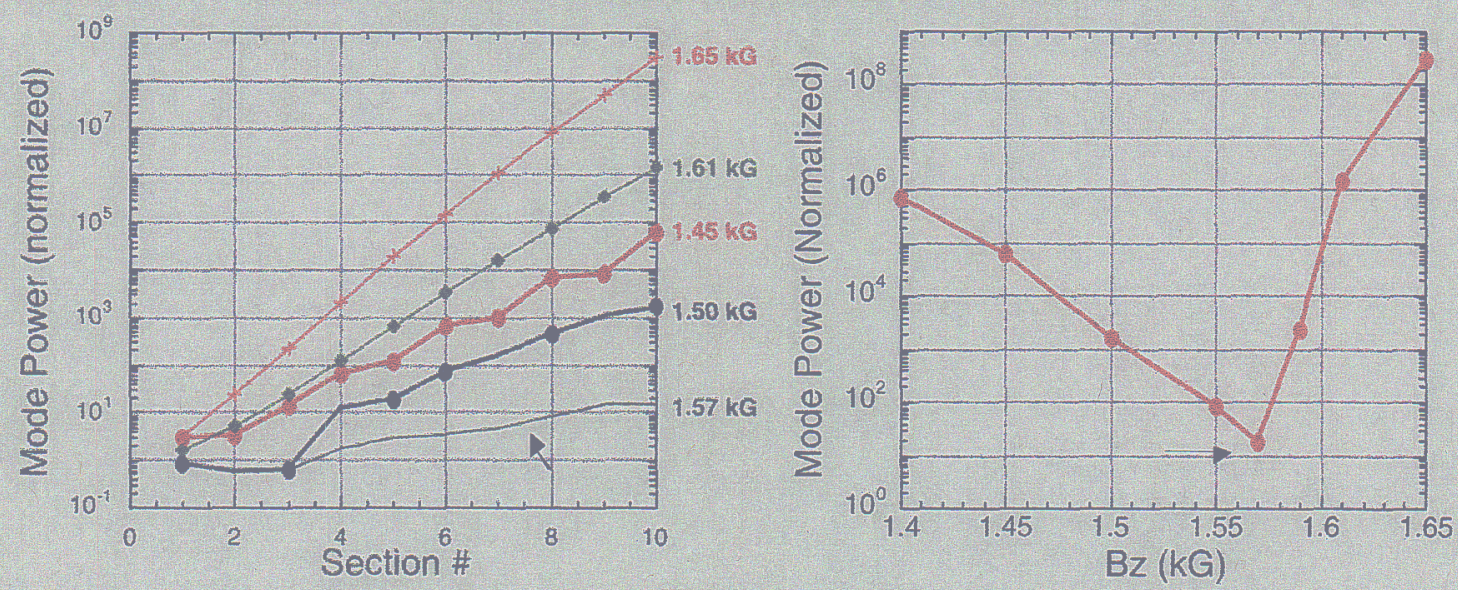

FIGURE 6. Computer predictions of BBU mode growth as a function of the number of sections (cavities) the beam has passed through for five values of the average solenoid field (left graph), and as a function of the average solenoid field (betatron phase advance) after 10 sections (right graph).

\section{SUMMARY}

The long-term goal of the RTA Facility is to build a prototype relativistic klystron that has all the major components required for a RK suitable for collider applications. The prototype would serve as a test bed for examining physics, engineering, and cost issues. The first major component, the $1-\mathrm{MeV}, 600-\mathrm{A}$, induction electron gun, of the prototype has been completed and commissioned. Before continuing with the next section of the prototype, we intend to perform a series of beam dynamics experiments. In particular, we will demonstrate the effectiveness of the Betatron Node Scheme. We are also continuing to study and optimize collider designs based on the RK-TBA scheme.

\section{ACKNOWLDEGEMENTS}

We thank Swapan Chattopadhyay, George Caporaso, Kim Robertson, and Simon Yu for their support and guidance. Dave Vanecek and Wayne Greenway provided invaluable mechanical engineering and technical services. John Corlett and Bob Rimmer designed the RF diagnostics. This work was performed under the auspices of the U.S. Department of Energy by University of California Lawrence Berkeley Livermore National Laboratory under contract No AC03-76SF00098 and Lawrence Livermore National Laboratory under contract No. W-7405-Eng-48.

\section{REFERENCES}

1. Sessler, A.M. and Yu, S.S., Phys. Rev. Letters 54, 889 (1987). 
2. Burns, M.J., et al., "DARHT Accelerator Update And Plans For Initial Operation", in Proceedings of IEEE 1999 Part. Accel. Conf., NY, 1999, pp. 617-621.

3. Houck, T.L. (ed.), et al., "Appendix A: A RF Power Source Upgrade to the NLC Based on the Relativistic Klystron Two-Beam Accelerator Concept," in the Zeroth Order Design Report for the Next Linear Collider, Stanford, Stanford Linear Accelerator Center, May 1996.

4. Westenskow, G.A. and Houck, T.L. "Relativistic Klystron Two-Beam Accelerator," IEEE Trans. Plasma Sci., 22, pp. $424-436$ (1994).

5. G.A. Westenskow and T.L. Houck, "Results of the Reacceleration Experiment: Experimental Study of the Relativistic Klystron Two-Beam Accelerator," in Proceedings of the 10th Int'l Conference on High Power Particle Beams, San Diego, CA (1994).

6. Lidia, S.M., et al., "Initial Commissioning Results of the RTA Injector", in Proceedings of IEEE 1999 Part. Accel. Conf., NY, 1999, pp. 3390-3392.

7. Lidia, S.M., et al., "RK-TBA Studies in Ka-Band", in Proceedings of XIX Intl. Linear Accel. Conf., IL, 1998, pp. 97-99.

8. Lefevre, T., et al., Phys. Rev. Letters 84, 1188-1191 (2000).

9. Godlove, T.F., "Induction Modulator for a Relativistic-Klystron Two-Beam Accelerator, Final Technical Report", FM Technolgies, Inc., Report No. 96-05-01, May 1996.

10. Sampayan, S.E., et al., "High-Performance Insulator Structures For Accelerator Applications", in Proceedings of IEEE 1997 Part. Accel. Conf., Vancouver, BC, 1997, pp. 1308-1310.

11. Neil, V.K., Hall, L.S., and Cooper, R.K., "Further Theoretical Studies of the Beam Breakup Instability", Particle Accelerators 9, pp. 213-222 (1979). 